\title{
The Distribution of Dissolved Methane and Its Air-Sea Flux in the Plume of a Seep Field, Lingtou Promontory, South China Sea
}

\author{
Pengfei Di $(\mathbb{D})^{1}$ Dong Feng $\left(\mathbb{D},{ }^{1,2,3}\right.$ and Duofu Chen $\mathbb{D}^{2,3}$ \\ ${ }^{1}$ CAS Key Laboratory of Ocean and Marginal Sea Geology, South China Sea Institute of Oceanology, Chinese Academy of Sciences, \\ Guangzhou, Guangdong 510301, China \\ ${ }^{2}$ Laboratory for Marine Mineral Resources, Qingdao National Laboratory for Marine Science and Technology, \\ Qingdao 266061, China \\ ${ }^{3}$ Shanghai Engineering Research Center of Hadal Science and Technology, College of Marine Sciences, Shanghai Ocean University, \\ Shanghai 201306, China
}

Correspondence should be addressed to Pengfei Di; pfd@scsio.ac.cn

Received 24 January 2019; Accepted 18 June 2019; Published 14 July 2019

Academic Editor: Giovanni Martinelli

Copyright (c) 2019 Pengfei Di et al. This is an open access article distributed under the Creative Commons Attribution License, which permits unrestricted use, distribution, and reproduction in any medium, provided the original work is properly cited.

\begin{abstract}
Methane $\left(\mathrm{CH}_{4}\right)$, the most abundant hydrocarbon gas in the atmosphere, plays an important role in global climate change. Quantifying the dissolved methane and its air-sea flux from hydrocarbon seeps is therefore of great importance. Large quantities of natural gas are emitted from the seafloor to the coastal ocean near the Lingtou Promontory, South China Sea. We quantified concentrations of methane in surface and bottom waters at 48 stations in a $56 \mathrm{~km}^{2}$ study area. High spatial variability in dissolved methane concentrations was observed in the surface mixed layer ( $0.5 \mathrm{~m}$ water depth) and bottom water (water-sediment interface), with values ranging from $2.90 \mathrm{nmol} \mathrm{L}^{-1}$ to $13570.02 \mathrm{nmol} \mathrm{L}^{-1}$ and from $4.98 \mathrm{nmol} \mathrm{L}$ to $31740.02 \mathrm{nmol} \mathrm{L}{ }^{-1}$, respectively. The significant difference between concentrations of dissolved methane in surface and bottom waters suggests that most of the methane emitted from the seafloor is dissolved in the water column. The dissolution of methane in seawater may result in local oxygen depletion that may lead to ecological effects. The $\delta^{13} \mathrm{C}$ values of dissolved methane ranging from $-59.76 \%$ to $-48.59 \%$ indicate a mixture of biogenic and thermogenic gas sources. The average air-sea methane flux of Yinggehai Basin was $672.57 \mu \mathrm{mol} \mathrm{m}{ }^{-2} \mathrm{~d}^{-1}$, which cannot be ignored in environment assessment. Coastal regions, especially with hydrocarbon seeps in shallow waters of the continental margin, may therefore be an important source of methane to the atmosphere.
\end{abstract}

\section{Introduction}

After water vapor and $\mathrm{CO}_{2}$, methane $\left(\mathrm{CH}_{4}\right)$ is the most important greenhouse gas in the atmosphere and plays an important role in global climate change. Methane is produced in oceanic sediments either by methanogens or through the breakdown of organic molecules $[1,2]$. Modern-day atmospheric methane concentrations of 1.77 ppmv (parts per million by volume) are more than twice the preindustrial value of $0.71 \mathrm{ppm}[3,4]$. Compared with carbon dioxide, methane is a more potent greenhouse gas, having a global warming potential that is 23 times higher on a $100 \mathrm{yr}$ timescale $[3,4]$. It is estimated that methane is released into the atmosphere worldwide at a rate of about 503-610 $\mathrm{Tg} \mathrm{yr}^{-1}$ [5]. Although hydrocarbon seeps release about 8-65 $\mathrm{Tg} \mathrm{yr}^{-1}$ of methane into the ocean [6], only $4-15 \mathrm{Tg} \mathrm{yr}^{-1}$ of methane is released into the atmosphere, accounting for about $1 \%-2 \%$ of the global atmospheric methane flux [7-12]. These values reveal that methane undergoes a complicated set of processes (involving both anaerobic and aerobic oxidation) in the ocean, with more than $85 \%$ of methane being consumed before reaching to the sea surface [13-16]. However, there is still substantial uncertainty surrounding estimates of the size and nature of methane sources and sinks and how their variations can affect atmospheric methane concentrations.

Natural hydrocarbon seeps in marine environments are widely distributed on the seabed of almost all continental margins, which are important sources of methane and other 
greenhouse gases to the ocean and the atmosphere $[9,17-$ 19]. Quantifying methane discharge from hydrocarbon seeps and its flux to the atmosphere is a major unsolved issue regarding the marine methane cycle. Hydrocarbon seeps may release methane dissolved in pore waters or, in the case of oversaturation, in the form of gas bubbles into the ocean $[9,17,18,20,21]$. When methane is emitted as gas bubbles, a fraction of the methane in the bubbles dissolves in the seawater via gas exchange during the bubbles' transport to the seawater surface, and the methane flux depends on release depth, bubble diameter, and the buoyancy force of the plume [22-24]. Methane can be released directly into the atmosphere from rising gas bubbles mainly in shallow waters $(<100 \mathrm{~m})$ while methane bubbles emitted from deep waters tend to become fully dissolved in the water column before reaching to the ocean surface. It has been shown that the flux and vertical transport of this methane are controlled by physical processes such as vertical mixing, turbulent diffusion, and upwelling $[24,25]$. Bubble dissolution leads to patches of higher methane concentrations in the water column (from tens of nmol L $\mathrm{L}^{-1}$ up to several $\mu \mathrm{mol} \mathrm{L}{ }^{-1}$ ), for example, in the Coal Oil Point seep field [19]. However, the dissolved methane flux arising from the dissolution of gas bubbles during transit through the water column or from the discharge of methane-rich pore fluids from the sediment into the water column is particularly difficult to quantify. The quantification of this indirect flux is critical for understanding the marine hydrocarbon contribution to the total atmospheric methane source.

Coastal regions are important sources of methane $(13 \mathrm{Tg}$ $\mathrm{CH}_{4} \mathrm{yr}^{-1}$ ) to the atmosphere $[5,26,27]$. Hundreds of hydrocarbon seeps are discovered in the coastal regions of Yinggehai Basin [28-30]. However, the distribution of dissolved methane and its air-sea fluxes has not been quantified. Sources and sinks of the seeping methane remain unclear. Here, we determined the distribution of dissolved methane from seawater samples and estimated the methane air-sea flux in the Lingtou Promontory seep area.

\section{Study Area}

The Yinggehai Basin, located to the southwest of Hainan Island $\left(16^{\circ} 50^{\prime}-20^{\circ} 00^{\prime} \mathrm{N}, 107^{\circ} 00-111^{\circ} 50^{\prime} \mathrm{E}\right)$, is one of the world's largest hydrocarbon seep regions [28, 31-33]. The basin is characterized by abundant hydrocarbon seeps, pockmarks, and mud volcanoes on the seabed [28, 31-33]. More than 120 hydrocarbon seeps have been found on the seabed at water depths of $<50 \mathrm{~m}$ along the eastern edge of the basin near the western coast of Hainan Island. At these seeps, the gas rises to the sea surface to form a nearshore bubble zone [28]. The total gas flux emitted from these hydrocarbon seeps to the water surface has been estimated as 294-956 $\mathrm{m}^{3} \mathrm{yr}^{-1}$ based on the displacement of water estimated using an inverted funnel [28], whereas a total methane flux of $4.84 \times 10^{4}$ to $6.84 \times 10^{4} \mathrm{~m}^{3} \mathrm{yr}^{-1}$ has been estimated for these seeps based on extrapolation of data obtained over $420 \mathrm{~h}$ using a gas flux measuring device on one hydrocarbon seep [29, 30, 34].

The Lingtou Promontory seep field is one of the most active areas in the Yinggehai Basin for natural hydrocarbon seepage [29, 30]. Over 20 perennial hydrocarbon seeps are emitting gases within an area measuring approximately 580 $\mathrm{m} \times 160 \mathrm{~m}$ located $\sim 300 \mathrm{~m}$ offshore Lingtou Promontory in Hainan Province, at water depths of 3-20 m (Figure 1(a)). It has been observed that gas bubbles (each up to $1-2 \mathrm{~cm}$ in diameter) are being emitted continuously from the hydrocarbon seeps on the seafloor (Figures 1(b) and 1(c)) and that these bubbles rise to the sea surface at about $10 \mathrm{~cm} \mathrm{~s}^{-1}$ and release their gases into the atmosphere $[29,30,35]$. Methane is released at rates ranging from $0.807 \times 10^{4}$ to $1.14 \times 10^{4}$ $\mathrm{m}^{3} \mathrm{yr}^{-1}$ from 20 hydrocarbon seeps in the Lingtou Promontory seep area, assuming that the hydrocarbon seeps have a similar gas flux $[29,30]$. However, this region has not been surveyed for dissolved methane distributions and air-sea fluxes to date.

\section{Methods}

On 19-20 August 2015, 96 seawater samples were sampled at 48 stations to quantify the dissolved methane concentration distribution along 4 west-east (W-E) transects from nearshore to the Yinggehai Basin (Figure 1(a) and Table 1). Sampling was carried out with a $5 \mathrm{~L}$ Niskin bottle coupled to a conductivity-temperature-depth (CTD) probe (RBR XR420) and a dissolved oxygen sensor (RBR) for the online monitoring of salinity, temperature, pressure, and dissolved oxygen (DO) concentrations in surface waters ( $0.5 \mathrm{~m}$ depth sampling level) and at the sea-sediment interface (bottom-water samples). The CTD sensors were calibrated to a temperature range of $2-35^{\circ} \mathrm{C}$ with a resolution of $0.002^{\circ} \mathrm{C}$, to a salinity range of $0-70 \mathrm{mS} \mathrm{cm}^{-1}$ with a resolution of $0.001 \mathrm{mS} \mathrm{cm}^{-1}$, and to a depth range of $0-6000 \mathrm{~m}$ with a resolution of $0.001 \mathrm{~m}$. The dissolved $\mathrm{O}_{2}$ sensor could detect within the range $0-500 \mu \mathrm{M}$ (saturation $0-120 \%$ ) with a resolution of $<1 \mu \mathrm{M}$ (saturation $0.4 \%$ ) [36].

All water samples, once collected, were stored in $122 \mathrm{~mL}$ crimp-top sample bottles. All sample bottles were flushed with two volumes of water and filled completely to eliminate bubbles. Two to three drops of saturated $\mathrm{HgCl}_{2}$ were added to the samples after collection, and the bottles were immediately capped with butyl rubber stoppers and crimp-sealed. To prevent the headspace gas from escaping through the rubber stopper, all bottles were stored stopper-side down until analysis. All samples were transported to the laboratory, and a $10 \mathrm{~mL} \mathrm{~N} \mathrm{~N}_{2}$ headspace was introduced into each bottle as described by Valentine et al. [37]. Two aliquots of the headspace were each analyzed for methane using a Wasson/HP complete gas composition analyzer at the Guangzhou Institute of Geochemistry, Chinese Academy of Science (CAS), Guangzhou, China. Replicate analyses of samples yielded a precision of $\pm 2 \%$ for samples with methane content. The methane concentration dissolved in seawater was calculated as described in Johnson et al. [38]:

$$
C_{\mathrm{L}}=C_{\mathrm{G}}\left(\frac{\beta R T}{22.356}+\frac{V_{\mathrm{G}}}{V_{\mathrm{L}}}\right),
$$

where $C_{\mathrm{L}}$ is the concentration of the gas to be measured in the liquid phase before equilibrium, $C_{\mathrm{G}}$ is the concentration 


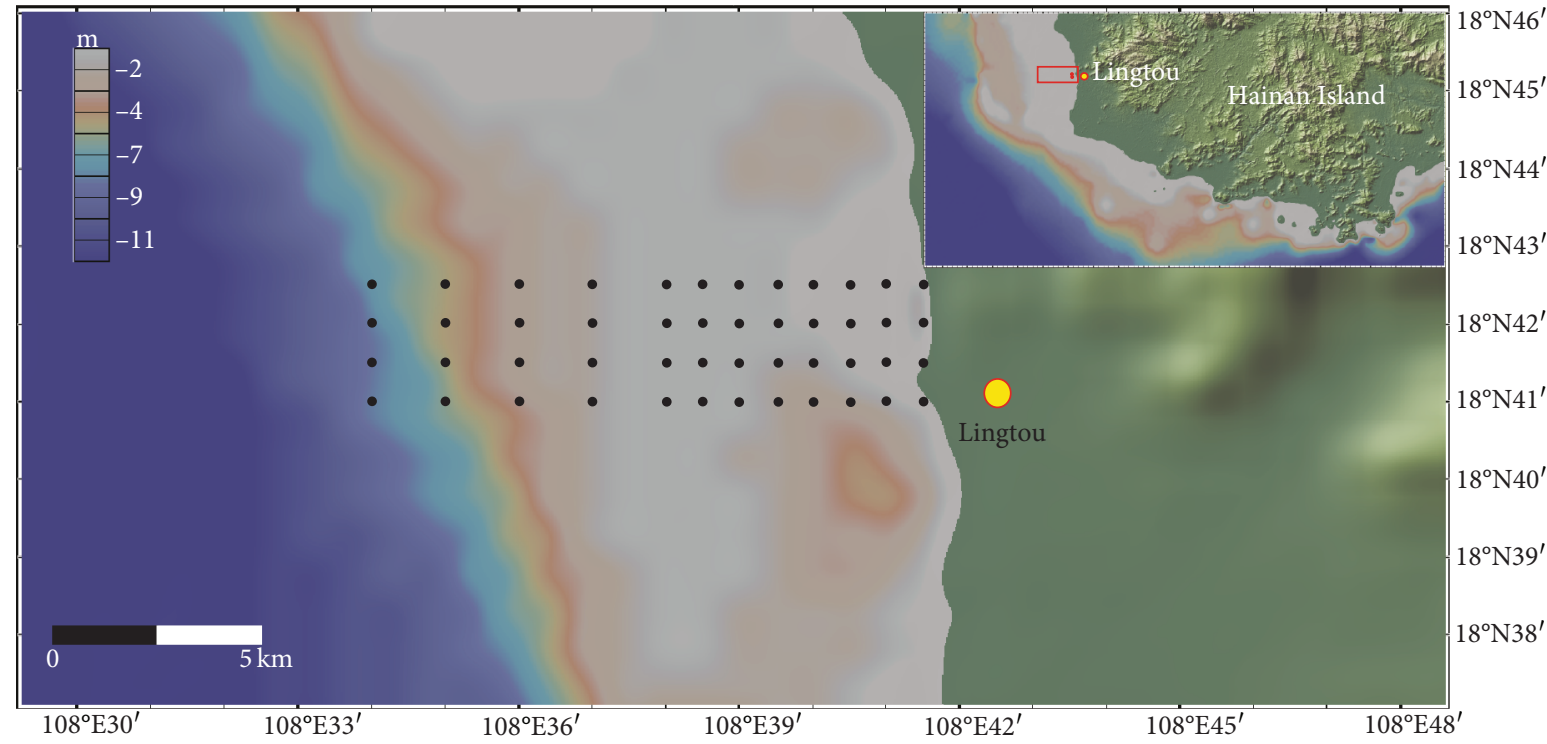

(a)

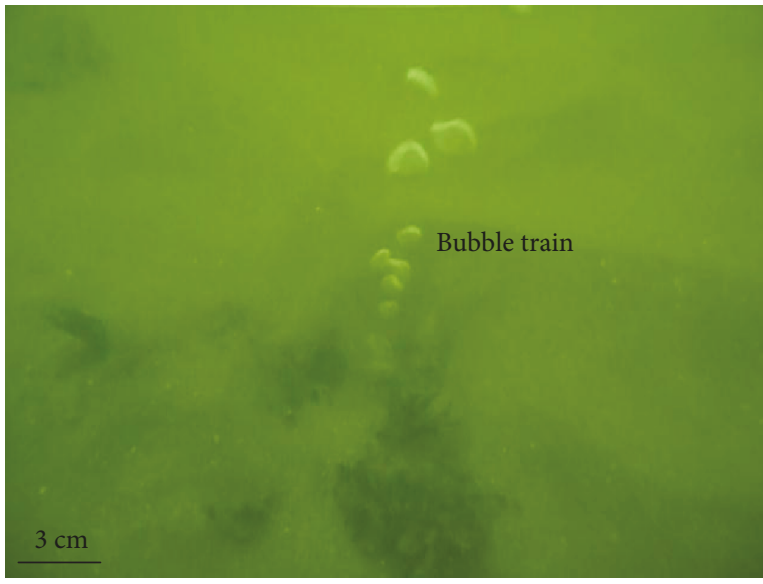

(b)

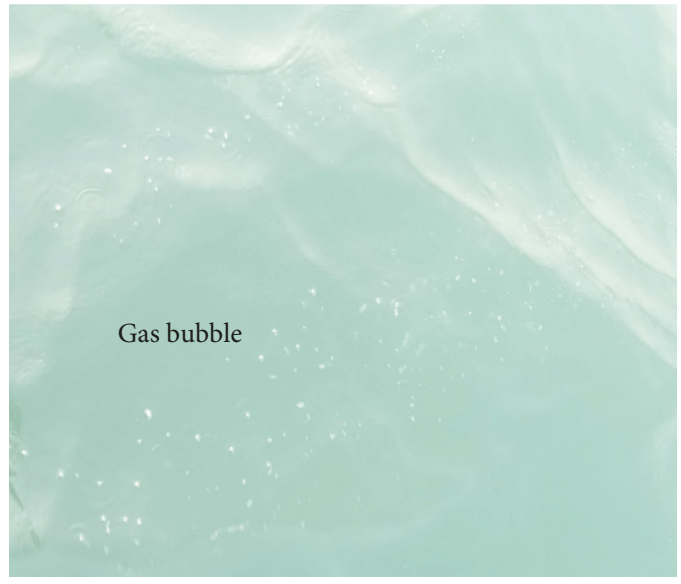

(c)

FIgure 1: (a) Bathymetric map of the hydrocarbon seep area near Lingtou Promontory. The red dots show the distribution of the nearshore hydrocarbon seeps, and the black dots are the seawater sample grid. (b) Photograph of rising irregular gas bubbles from a seep site. (c) Gas bubbles at the sea surface.

of the gas to be measured in the gas phase after equilibrium, $\beta$ is the Bunsen solubility of the gas to be measured ( $\mathrm{L} \mathrm{L} \mathrm{L}^{-1}$ atm. $^{-1}$ ), $R$ is the gas constant ( $\mathrm{L}$ atm. $\mathrm{mol}^{-1} \mathrm{~K}^{-1}$ ), and 22.356 is the molecular volume of methane $\left(\mathrm{L} \mathrm{mol}{ }^{-1}\right)$.

Seawater samples with high dissolved methane concentrations were analyzed to determine the stable carbon isotope composition of methane. Stable carbon isotope analyses were conducted using an isotope ratio mass spectrometer at the Guangzhou Institute of Geochemistry, CAS, Guangzhou, China. All isotopic ratios are presented in the $\delta$ notation with respect to the Vienna Pee Dee Belemnite (VPDB) standard and have an analytical error of $<1 \%$.

The air-sea methane flux $(F)$ was calculated as described in Leifer and MacDonald [39]:

$$
F=K_{w}\left(C_{w}-C_{a}\right)
$$

where $K_{w}$ is the transfer velocity for methane at in situ temperatures $\left(\mathrm{cm} \mathrm{hr}^{-1}\right), C_{w}$ is the measured dissolved methane concentration in seawater (nM), and $C_{a}$ is the airequilibrated seawater methane concentration (nM), which was calculated for in situ temperatures and salinities using the Bunsen solubility data of Wiesenburg and Guinasso [40]. We have assumed, for these calculations, an atmospheric methane mixing ratio of $1.80 \mathrm{ppmv}$, which in turn assumes an annual increase of $\sim 7 \mathrm{ppbv}$ [3]. The resulting flux is given in units of $\mu \mathrm{mol} \mathrm{m}{ }^{-2} \mathrm{~d}^{-1}$. The determination of the transfer velocity, $K_{w}$, has been thoroughly discussed in Wanninkhof et al. [41]. For our purposes, we used the following relationship, as suggested by Wanninkhof [42], for wind speeds of $<15 \mathrm{~m} \mathrm{~s}^{-1}$ :

$$
K_{w}=0.24 * U^{2}\left(\frac{\mathrm{Sc}_{\mathrm{CH}_{4}}}{660}\right)^{-0.5}
$$


TABLE 1: The $\mathrm{CH}_{4}$ concentrations of bottom and surface seawater, as well as the $\mathrm{O}_{2}$ concentration, temperature, water depth, salinity of all the sites, and the $\delta^{13} \mathrm{C}_{\mathrm{CH}_{4}}$ values in seawater and bubbles from the Lingtou Promontory seep area.

\begin{tabular}{|c|c|c|c|c|c|c|c|c|}
\hline Station & Latitude & Longitude & $\begin{array}{l}\text { Water depth } \\
(\mathrm{m})\end{array}$ & $\begin{array}{c}\mathrm{CH}_{4} \\
\text { concentration } \\
\left(\mathrm{nmol} \mathrm{L}^{-1}\right)\end{array}$ & $\begin{array}{l}\delta^{13} \mathrm{C} \\
(\% 0)\end{array}$ & $\begin{array}{c}\mathrm{O}_{2} \text { concentration } \\
(\mathrm{mL})\end{array}$ & $\begin{array}{l}\text { Salinity } \\
\text { (\%o) }\end{array}$ & $\begin{array}{c}\text { Temperature } \\
\left({ }^{\circ} \mathrm{C}\right)\end{array}$ \\
\hline \multirow{2}{*}{ Dot 1} & \multirow{2}{*}{$18^{\circ} 41^{\prime} 52^{\prime \prime}$} & \multirow{2}{*}{$108^{\circ} 41^{\prime} 13^{\prime \prime}$} & 0.50 & 3.18 & - & 7.81 & 32.72 & 29.67 \\
\hline & & & 4.10 & 10.00 & - & 8.13 & 32.62 & 29.38 \\
\hline \multirow{2}{*}{ Dot 2} & \multirow{2}{*}{$18^{\circ} 41^{\prime} 37^{\prime \prime}$} & \multirow{2}{*}{$108^{\circ} 40^{\prime} 29^{\prime \prime}$} & 0.50 & 11.27 & - & 7.57 & 33.14 & 29.37 \\
\hline & & & 5.31 & 5472.08 & - & 7.82 & 33.21 & 29.16 \\
\hline \multirow{2}{*}{ Dot 3} & \multirow{2}{*}{$18^{\circ} 41^{\prime} 58^{\prime \prime}$} & \multirow{2}{*}{$108^{\circ} 39^{\prime} 30^{\prime \prime}$} & 0.50 & 7.19 & - & 7.42 & 33.41 & 29.21 \\
\hline & & & 6.48 & 9.93 & - & 7.91 & 33.38 & 29.13 \\
\hline \multirow{2}{*}{ Dot 4} & \multirow{2}{*}{$18^{\circ} 41^{\prime} 58^{\prime \prime}$} & \multirow{2}{*}{$108^{\circ} 38^{\prime} 57^{\prime \prime}$} & 0.50 & 12.09 & - & 7.49 & 33.48 & 28.92 \\
\hline & & & 7.10 & 11.06 & - & 7.94 & 33.37 & 29.00 \\
\hline \multirow{2}{*}{ Dot 5} & \multirow{2}{*}{$18^{\circ} 42^{\prime} 02^{\prime \prime}$} & \multirow{2}{*}{$108^{\circ} 38^{\prime} 28^{\prime \prime}$} & 0.50 & 19.74 & - & 7.31 & 33.49 & 29.12 \\
\hline & & & 7.50 & 23.80 & - & 7.38 & 33.33 & 29.09 \\
\hline \multirow{2}{*}{ Dot 6} & \multirow{2}{*}{$18^{\circ} 42^{\prime} 01^{\prime \prime}$} & \multirow{2}{*}{$108^{\circ} 37^{\prime} 51^{\prime \prime}$} & 0.50 & 36.10 & - & 7.59 & 33.43 & 28.82 \\
\hline & & & 8.07 & 10.95 & - & 7.49 & 33.26 & 28.61 \\
\hline \multirow{2}{*}{ Dot 7} & \multirow{2}{*}{$18^{\circ} 42^{\prime} 02^{\prime \prime}$} & \multirow{2}{*}{$108^{\circ} 37^{\prime} 01^{\prime \prime}$} & 0.50 & 19.55 & - & 7.43 & 33.39 & 28.84 \\
\hline & & & 7.30 & 4.98 & - & 7.63 & 33.35 & 28.71 \\
\hline \multirow{2}{*}{ Dot 8} & $18^{\circ} 41^{\prime} 57^{\prime \prime}$ & $108^{\circ} 35^{\prime} 58^{\prime \prime}$ & 0.50 & 64.70 & - & 7.48 & 33.36 & 28.74 \\
\hline & & & 5.34 & 28.54 & - & 7.82 & 33.24 & 28.74 \\
\hline Det 9 & $18^{\circ} 41^{\prime} 58^{\prime \prime}$ & $108^{\circ} 35^{\prime} 00^{\prime \prime}$ & 0.50 & 9.65 & - & 7.39 & 33.39 & 28.71 \\
\hline Dot 9 & 184158 & 1083500 & 6.30 & 26.31 & - & 7.64 & 33.32 & 28.68 \\
\hline & $18^{\circ} 42^{\prime} 01^{\prime \prime}$ & $108^{\circ}$ & 0.50 & 68.95 & - & 6.94 & 33.36 & 28.86 \\
\hline Dot 10 & 184201 & 1083400 & 7.67 & 9.13 & - & 7.23 & 33.36 & 28.78 \\
\hline Dot 11 & $18^{\circ} 42^{\prime} 27^{\prime \prime}$ & $108^{\circ} 34^{\prime} 02^{\prime \prime}$ & 0.50 & 7.62 & - & 7.01 & 33.37 & 28.96 \\
\hline DOt 11 & 184227 & 1083402 & 6.41 & 79.85 & - & 7.47 & 33.36 & 28.81 \\
\hline & $18^{\circ} 42^{\prime} 27^{\prime \prime}$ & $108^{\circ} 35^{\prime} 01^{\prime \prime}$ & 0.50 & 1500.49 & - & 7.29 & 33.39 & 28.90 \\
\hline Dot 12 & 184227 & 1083501 & 6.55 & 17.73 & - & 7.27 & 33.27 & 28.88 \\
\hline & $18^{\circ} 42^{\prime} 32^{\prime \prime}$ & $108^{\circ}$ & 0.50 & 975.45 & - & 6.85 & 33.47 & 29.21 \\
\hline Dot 13 & 184232 & 1083600 & 5.22 & 50.36 & - & 7.19 & 33.39 & 29.05 \\
\hline Dot 14 & $18^{\circ} 42^{\prime} 43^{\prime \prime}$ & $108^{\circ} 38^{\prime} 31^{\prime \prime}$ & 0.50 & 11.43 & - & 7.02 & 33.41 & 29.42 \\
\hline DOt 14 & 184243 & 1083831 & 5.94 & 7.35 & - & 7.23 & 33.32 & 29.31 \\
\hline & & & 0.50 & 12.45 & - & 6.93 & 33.4 & 29.27 \\
\hline Dot 15 & 184228 & 1083835 & 7.36 & 45.40 & - & 7.15 & 33.38 & 29.23 \\
\hline & $18^{\circ} 42^{\prime} 25^{\prime \prime}$ & $108^{\circ} 39^{\prime} 02^{\prime \prime}$ & 0.50 & 10.56 & & 7.17 & 33.38 & 29.28 \\
\hline Dot 16 & 184225 & 1083902 & 6.11 & 11.54 & & 7.41 & 33.33 & 29.21 \\
\hline Dot 17 & $18^{\circ} 42^{\prime} 24^{\prime \prime}$ & $108^{\circ} 39^{\prime} 34^{\prime \prime}$ & 0.50 & 6.80 & - & 6.96 & 33.36 & 29.32 \\
\hline Dot $1 /$ & 184224 & 1083934 & 7.38 & 65.62 & - & 7.13 & 33.38 & 29.28 \\
\hline & & $100^{\circ} 20^{\prime} 0^{\prime \prime}$ & 0.50 & 26.09 & - & 7.04 & 33.35 & 29.47 \\
\hline Dot 18 & $18^{\circ} 42^{\prime} 33^{\prime \prime}$ & $108^{-3} 39^{\prime} 59^{\prime \prime}$ & 6.79 & 22.54 & - & 7.26 & 33.31 & 29.45 \\
\hline Dot 19 & $18^{\circ} 42^{\prime} 30^{\prime \prime}$ & $108^{\circ} 40^{\prime} 31^{\prime \prime}$ & 0.50 & 4140.60 & - & 7.12 & 33.23 & 29.68 \\
\hline & & 1084031 & 6.42 & 17.98 & - & 7.38 & 32.98 & 29.66 \\
\hline Dot 20 & $18^{\circ} 42^{\prime} 35^{\prime \prime}$ & $108^{\circ} 41^{\prime} 05^{\prime \prime}$ & 0.50 & 16.67 & - & 7.18 & 33.17 & 30.04 \\
\hline Dot 20 & & 1084105 & 5.48 & 9.01 & - & 7.67 & 33.15 & 30.02 \\
\hline & & & 0.50 & 19.15 & - & 7.22 & 33.17 & 29.98 \\
\hline Dot 21 & 184159 & $108^{\circ} 41^{\prime} 04^{\prime \prime}$ & 4.94 & 9.42 & - & 7.85 & 33.22 & 29.93 \\
\hline Dot 22 & $18^{\circ} 41^{\prime} 59^{\prime \prime}$ & $108^{\circ} 41^{\prime} 29^{\prime \prime}$ & 0.50 & 14.95 & - & 7.58 & 32.96 & 30.32 \\
\hline Dot 22 & 184159 & 1084129 & 4.7 & 390.64 & - & 8.08 & 32.93 & 30.31 \\
\hline
\end{tabular}


TABle 1: Continued.

\begin{tabular}{|c|c|c|c|c|c|c|c|c|}
\hline Station & Latitude & Longitude & $\begin{array}{l}\text { Water depth } \\
(\mathrm{m})\end{array}$ & $\begin{array}{c}\mathrm{CH}_{4} \\
\text { concentration } \\
\left(\mathrm{nmol} \mathrm{L}^{-1}\right)\end{array}$ & $\begin{array}{l}\delta^{13} \mathrm{C} \\
(\% \circ)\end{array}$ & $\begin{array}{c}\mathrm{O}_{2} \text { concentration } \\
(\mathrm{mL})\end{array}$ & $\begin{array}{l}\text { Salinity } \\
(\% 0)\end{array}$ & $\begin{array}{c}\text { Temperature } \\
\left({ }^{\circ} \mathrm{C}\right)\end{array}$ \\
\hline \multirow{2}{*}{ Dot 23} & \multirow{2}{*}{$18^{\circ} 40^{\prime} 59^{\prime \prime}$} & \multirow{2}{*}{$108^{\circ} 40^{\prime} 59^{\prime \prime}$} & 0.50 & 12318.50 & -59.17 & 7.15 & 33.04 & 29.55 \\
\hline & & & 3.44 & 2313.53 & - & 7.23 & 33.08 & 29.49 \\
\hline \multirow{2}{*}{ Dot 24} & \multirow{2}{*}{$\begin{array}{l}18^{\circ} 41^{\prime} \\
59.5^{\prime \prime}\end{array}$} & \multirow{2}{*}{$108^{\circ} 40^{\prime} 29^{\prime \prime}$} & 0.50 & 45.89 & - & 7.2 & 33.34 & 29.29 \\
\hline & & & 4.45 & 31740.02 & -59.54 & 7.36 & 33.34 & 29.28 \\
\hline \multirow{2}{*}{ Dot 25} & \multirow{2}{*}{$18^{\circ} 40^{\prime} 59^{\prime \prime}$} & \multirow{2}{*}{$108^{\circ} 40^{\prime} 03^{\prime \prime}$} & 0.50 & 13577.35 & -59.76 & 7.26 & 33.34 & 29.23 \\
\hline & & & 4.45 & - & - & 7.39 & 33.33 & 29.22 \\
\hline \multirow{2}{*}{ Dot 26} & \multirow{2}{*}{$18^{\circ} 40^{\prime} 56^{\prime \prime}$} & \multirow{2}{*}{$108^{\circ} 39^{\prime} 15^{\prime \prime}$} & 0.5 & 33.25 & - & 7.16 & 33.37 & 29.19 \\
\hline & & & 3.72 & 209.05 & - & 7.12 & 33.38 & 29.16 \\
\hline \multirow{2}{*}{ Dot 27} & \multirow{2}{*}{$18^{\circ} 40^{\prime} 59^{\prime \prime}$} & \multirow{2}{*}{$108^{\circ} 38^{\prime} 59^{\prime \prime}$} & 0.5 & 171.06 & - & 7.18 & 33.39 & 29.22 \\
\hline & & & 5.55 & 10.04 & - & 7.2 & 33.23 & 29.17 \\
\hline \multirow{2}{*}{ Dot 28} & \multirow{2}{*}{$\begin{array}{l}18^{\circ} 40^{\prime} \\
59.9^{\prime \prime}\end{array}$} & \multirow{2}{*}{$108^{\circ} 38^{\prime} 29^{\prime \prime}$} & 0.5 & 12.23 & - & 7.12 & 33.38 & 29.31 \\
\hline & & & 6.08 & 435.28 & - & 7.22 & 33.34 & 29.23 \\
\hline \multirow{2}{*}{ Dot 29} & \multirow{2}{*}{$\begin{array}{l}18^{\circ} 40^{\prime} \\
59.5^{\prime \prime}\end{array}$} & \multirow{2}{*}{$108^{\circ} 37^{\prime} 59^{\prime \prime}$} & 0.5 & 8.84 & - & 7.17 & 33.38 & 29.22 \\
\hline & & & 7.01 & 8.69 & - & 7.26 & 33.29 & 29.12 \\
\hline \multirow{2}{*}{ Dot 30} & $18^{\circ} 40^{\prime}$ & $108^{\circ} 36^{\prime} 58^{\prime \prime}$ & 0.5 & 107.98 & - & 7.2 & 33.39 & 28.68 \\
\hline & $59.3^{\prime \prime}$ & 1083658 & 7.03 & 22.35 & - & 7.19 & 33.33 & 28.65 \\
\hline & $18^{\circ} 40^{\prime}$ & $108^{\circ} 35^{\prime}$ & 0.5 & 6.17 & - & 7.15 & 33.39 & 28.71 \\
\hline Dot 31 & $59.7^{\prime \prime}$ & $58.2^{\prime \prime}$ & 5.74 & 23386.77 & -48.59 & 7.2 & 33.25 & 28.66 \\
\hline Dot 32 & $18^{\circ} 41^{\prime} 01^{\prime \prime}$ & $108^{\circ} 34^{\prime}$ & 0.50 & 2.90 & - & 7.09 & 33.37 & 29.03 \\
\hline 00032 & & $59.4^{\prime \prime}$ & 5.75 & 9.99 & - & 7.25 & 33.27 & 28.87 \\
\hline & $18^{\circ} 40^{\prime}$ & 58 & 0.50 & 22.08 & - & 7.08 & 33.34 & 28.93 \\
\hline Dot 33 & $59.7^{\prime \prime}$ & 1083358 & 6.96 & 5.17 & - & 7.2 & 33.32 & 28.75 \\
\hline Dot 34 & $18^{\circ} 41^{\prime}$ & $108^{\circ} 34^{\prime} 00^{\prime \prime}$ & 0.50 & 9.80 & - & 7.04 & 33.35 & 29.05 \\
\hline 10034 & $31.6^{\prime \prime}$ & & 10.38 & 171.94 & - & 7.29 & 33.12 & 28.79 \\
\hline Det 35 & $18^{\circ} 41^{\prime}$ & $108^{\circ} 35^{\prime} 01^{\prime \prime}$ & 0.50 & 115.34 & - & 7.14 & 33.36 & 29.07 \\
\hline Dot 35 & $29.4^{\prime \prime}$ & 1083501 & 9.76 & 107.83 & - & 7.3 & 33.32 & 29.04 \\
\hline Dot 36 & $18^{\circ} 41^{\prime}$ & $108^{\circ} 36^{\prime} 02^{\prime \prime}$ & 0.50 & 7.82 & - & 7.08 & 33.39 & 28.86 \\
\hline & $28.6^{\prime \prime}$ & & 6.52 & 101.58 & - & 7.23 & 33.28 & 28.85 \\
\hline Dot 37 & $18^{\circ} 41^{\prime}$ & $108^{\circ} 37^{\prime} 01^{\prime \prime}$ & 0.50 & 8.51 & - & 7.18 & 33.45 & 28.94 \\
\hline 00131 & $28.9^{\prime \prime}$ & 1083701 & 5.27 & 547.26 & - & 7.22 & 33.3 & 28.81 \\
\hline Det 38 & $18^{\circ} 41^{\prime}$ & $108^{\circ}$ & 0.50 & 4.01 & - & 7.07 & 33.47 & 29.53 \\
\hline Dot 38 & $29.8^{\prime \prime}$ & 1083801 & 6.70 & 573.54 & - & 7.17 & 33.35 & 29.14 \\
\hline Dot 39 & $18^{\circ} 41^{\prime}$ & $108^{\circ} 38^{\prime}$ & 0.5 & 7.69 & - & 7.04 & 33.43 & 29.57 \\
\hline Dot 39 & $29.9^{\prime \prime}$ & $38.6^{\prime \prime}$ & 8.21 & 4.98 & - & 7.24 & 33.34 & 29.15 \\
\hline Det 40 & $18^{\circ} 41^{\prime}$ & $108^{\circ} 39^{\prime} 01^{\prime \prime}$ & 0.5 & 250.93 & - & 7.14 & 33.65 & 29.49 \\
\hline Dot 40 & $29.8^{\prime \prime}$ & 1083901 & 8.06 & 162.68 & - & 7.29 & 33.59 & 29.19 \\
\hline Dot 41 & $18^{\circ} 41^{\prime}$ & $108^{\circ} 39^{\prime}$ & 0.5 & 24.02 & - & 7.01 & 33.66 & 29.49 \\
\hline Dot 41 & $29.3^{\prime \prime}$ & $30.7^{\prime \prime}$ & 7.59 & 9.10 & - & 7.18 & 33.64 & 29.18 \\
\hline Det 42 & $18^{\circ} 41^{\prime} 29^{\prime \prime}$ & $108^{\circ} 40^{\prime} 01^{\prime \prime}$ & 0.5 & 7.75 & - & 7.24 & 33.35 & 29.56 \\
\hline 10042 & & 1004001 & 6.99 & 65.26 & - & 7.35 & 33.37 & 29.41 \\
\hline & $18^{\circ} 41^{\prime}$ & $108^{\circ} 40^{\prime}$ & 0.5 & 4.13 & - & 7.33 & 33.44 & 29.75 \\
\hline Dot 43 & $28.4^{\prime \prime}$ & $31.1^{\prime \prime}$ & 6.37 & 233.55 & - & 7.42 & 33.34 & 29.65 \\
\hline Dot 44 & $18^{\circ} 41^{\prime}$ & $108^{\circ} 39^{\prime}$ & 0.5 & 28.80 & - & 7.1 & 33.8 & 29.59 \\
\hline Dot 44 & $58.4^{\prime \prime}$ & $59.7^{\prime \prime}$ & 5.81 & 21.07 & - & 7.38 & 33.72 & 29.53 \\
\hline
\end{tabular}


TABle 1: Continued.

\begin{tabular}{|c|c|c|c|c|c|c|c|c|}
\hline Station & Latitude & Longitude & $\begin{array}{l}\text { Water depth } \\
(\mathrm{m})\end{array}$ & $\begin{array}{c}\mathrm{CH}_{4} \\
\text { concentration } \\
\left(\mathrm{nmol} \mathrm{L}^{-1}\right)\end{array}$ & $\begin{array}{l}\delta^{13} \mathrm{C} \\
(\%)\end{array}$ & $\begin{array}{l}\mathrm{O}_{2} \text { concentration } \\
(\mathrm{mL})\end{array}$ & $\begin{array}{l}\text { Salinity } \\
(\% 0)\end{array}$ & $\begin{array}{c}\text { Temperature } \\
\left({ }^{\circ} \mathrm{C}\right)\end{array}$ \\
\hline \multirow{2}{*}{ Dot 45} & \multirow{2}{*}{$\begin{array}{l}18^{\circ} 41^{\prime} \\
31.2^{\prime \prime}\end{array}$} & \multirow{2}{*}{$\begin{array}{c}108^{\circ} 41^{\prime} \\
11.8^{\prime \prime}\end{array}$} & 0.5 & 153.62 & - & 7.46 & 33.16 & 30.09 \\
\hline & & & 6.15 & 122.29 & - & 7.93 & 33.14 & 29.98 \\
\hline \multirow{2}{*}{ Dot 46} & \multirow{2}{*}{$\begin{array}{l}18^{\circ} 40^{\prime} \\
54.6^{\prime \prime}\end{array}$} & \multirow{2}{*}{$108^{\circ} 41^{\prime} 04^{\prime \prime}$} & 0.5 & 551.39 & - & 7.72 & 33.15 & 30.2 \\
\hline & & & 5.23 & 495.30 & - & 8.12 & 33.12 & 30.12 \\
\hline \multirow{2}{*}{ Dot 47} & \multirow{2}{*}{$\begin{array}{l}18^{\circ} 40^{\prime} \\
58.7^{\prime \prime}\end{array}$} & \multirow{2}{*}{$\begin{array}{c}108^{\circ} 41^{\prime} \\
29.2^{\prime \prime}\end{array}$} & 0.5 & 7.73 & - & 7.9 & 32.86 & 30.42 \\
\hline & & & 5.35 & 169.94 & - & 8.02 & 32.76 & 30.25 \\
\hline \multirow{2}{*}{ Dot 48} & \multirow{2}{*}{$\begin{array}{l}18^{\circ} 41^{\prime} \\
32.4^{\prime \prime}\end{array}$} & \multirow{2}{*}{$\begin{array}{l}108^{\circ} 41^{\prime} \\
35.4^{\prime \prime}\end{array}$} & 0.5 & 19.05 & - & 7.89 & 32.79 & 30.49 \\
\hline & & & 3.45 & 187.42 & - & 8.32 & 32.61 & 30.41 \\
\hline \multirow{2}{*}{ Dot 49} & \multirow{2}{*}{$18^{\circ} 42^{\prime} 0.2^{\prime \prime}$} & \multirow{2}{*}{$\begin{array}{c}108^{\circ} 41^{\prime} \\
28.1^{\prime \prime}\end{array}$} & 0.5 & 14.00 & - & 8.05 & 32.82 & 30.49 \\
\hline & & & 3.6 & 11.92 & - & 8.59 & 32.7 & 30.3 \\
\hline $\mathrm{YG}^{*}$ & $\begin{array}{l}18^{\circ} 41^{\prime} \\
15.7^{\prime \prime}\end{array}$ & $\begin{array}{l}108^{\circ} 41^{\prime} \\
37.0^{\prime \prime}\end{array}$ & 5.00 & - & -35.98 & - & - & - \\
\hline Ys- $1^{*}$ & $18^{\circ} 41.136^{\prime}$ & $108^{\circ} 41.304^{\prime}$ & 0.50 & - & -35.51 & - & - & - \\
\hline
\end{tabular}

*Data are taken from Huang et al. [28] and Di et al. [35].

where $U$ is the average wind speed at $10 \mathrm{~m}$ above the water surface at the time of measurement and $\mathrm{Sc}_{\mathrm{CH}_{4}}$ was determined from Wanninkhof [42] using

$$
\mathrm{Sc}_{\mathrm{CH}_{4}}=A+B t+C t^{2}+D t^{3}+E t^{4}
$$

where $t$ is the in situ temperature $\left({ }^{\circ} \mathrm{C}\right)$. Values for constants $A$ (2102.2), B (-131.54), $C$ (4.4931), D (-0.08676), and $E$ $(0.00070663)$ were taken from Wanninkhof [42].

Average wind speed data were measured using a revolving vane anemometer (GM8901, range: $0.3-45 \mathrm{~m} \mathrm{~s}^{-1}$ ) at $0.5 \mathrm{~m}$ above sea level on-board and corrected to a standard height of $10 \mathrm{~m}$ by applying the following power law:

$$
\frac{V_{h}}{V_{10}}=\left(\frac{h}{10}\right)^{0.13}
$$

where $V_{h}$ is the mean wind speed at a height of $h, V_{10}$ is the mean wind speed at $10 \mathrm{~m}$, and $h$ is the effective height of the anemometer above the mean sea level. The air-sea flux was calculated for all the surface water samples. Owing to the potential inaccuracy of wind speed measurements and the parameterizations of the gas transfer velocity, there may be uncertainties surrounding the air-sea flux data.

\section{Results and Discussion}

4.1. Concentrations of Dissolved Methane. Methane concentrations for the 96 seawater samples collected at the Lingtou Promontory seep area are all above $\sim 2 \mathrm{nmol} \mathrm{L}^{-1}$ of the atmospheric equilibrium concentration (Table 1) [43]. The methane concentrations of surface seawater samples range from $2.90 \mathrm{nmol} \mathrm{L}^{-1}$ to an anomalously high value of $13577.35 \mathrm{nmol} \mathrm{L}^{-1}$ close to the hydrocarbon seep sites (Figure 2(a)). The average concentration for the surface seawater samples is $704.40 \mathrm{nmol} \mathrm{L}^{-1}$, which is much higher than the maximal value in surface waters $\left(\sim 1800 \mathrm{nmol} \mathrm{L}^{-1}\right)$ measured in the Santa Barbara Channel (Coal Oil Point), one of the most active known hydrocarbon seep areas in the world [39]. The methane concentrations of the bottomwater samples range from $4.98 \mathrm{nmol} \mathrm{L}^{-1}$ to $31740.02 \mathrm{nmol} \mathrm{L}^{-1}$ with an average value of $1376.75 \mathrm{nmol} \mathrm{L}^{-0.1}$. The dissolved methane concentration data for the bottom-water samples reveal a distinct gas plume situated at the seafloor (Figure 2(b)). The anomalously high concentrations of methane dissolved in bottom waters are higher than the surface methane concentrations but much lower than the maximum methane concentration $\left(2500 \mu \mathrm{mol} \mathrm{L}^{-1}\right)$ measured by the methane sensor at the hydrocarbon seep sites [29] as well as the calculated saturated methane concentration $\left(1413 \mu \mathrm{ML}^{-1}\right)$ for conditions of $29^{\circ} \mathrm{C}, 0.538 \mathrm{~mol} \mathrm{~kg}^{-1}$ salinity, and $1.26 \mathrm{bar}$ pressure [44]. Similar results have been reported in other gas seepage areas, such as the North Sea, south of the Dogger Bank, and Tommeliten, the central North Sea [45]. The difference value of the dissolved methane concentrations between surface waters and bottom waters may be attributed to removal by microbial methane oxidation and lateral dispersion by physical transport, favored by strong tidal currents and ocean current [43, 45-47].

Exceptionally high methane concentrations usually point to hydrocarbon seepage. Measurements of methane concentration can be used to detect a gas plume situated near the seafloor, and the transect profiles can reveal discrete maxima that indicate whether the methane released from gas bubbles was emitted from the hydrocarbon seeps or from a horizon where methane from different sources converge. We divided our data into four transects from north to south along the shore. Along the fourth transect, two stations with extremely high methane concentrations were found to be associated with locations of intense hydrocarbon seepage at water depths of 3-20 m (Figure 3). One station with high methane 

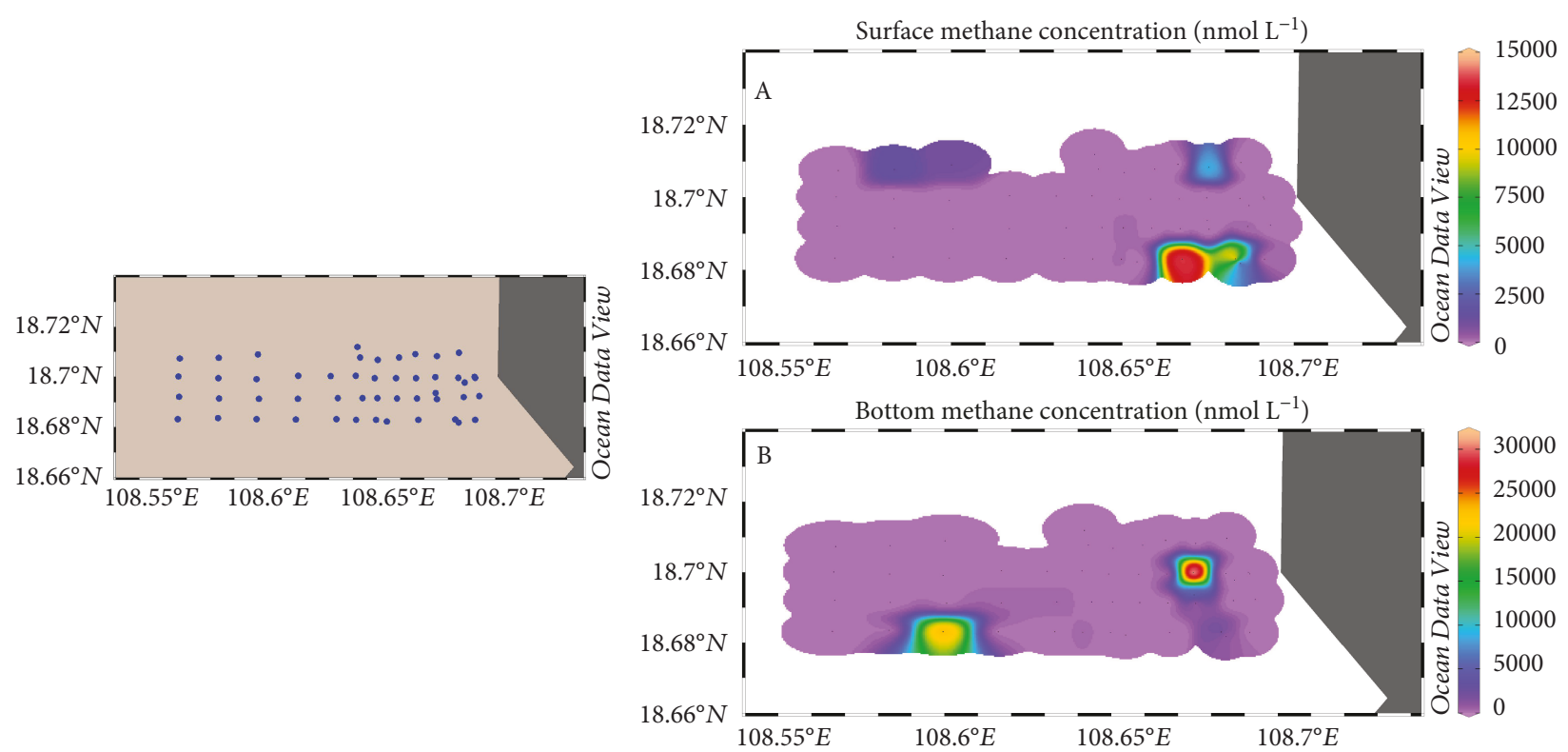

Figure 2: The distribution of dissolved methane concentrations in bottom waters and surface waters, respectively. The plot was generated using the Ocean Data View Version 4.5.7 (https://odv.awi.de) gridding weighted averages.

concentrations is close to the shore of the Lingtou Promontory at the surface, which indicates that methane was released from a horizon where different sources of methane converge. The other station with high methane concentrations is far offshore and in bottom waters, which indicates that methane was released from gas bubbles emitted from the hydrocarbon seeps. The high methane concentrations in bottom waters along the second and third transects, which eventually decrease offshore (Figure 3), indicate that methane is released from seafloor seeps. Surface methane concentrations were mostly in equilibrium with atmospheric concentrations along the second and third transects. Dissolved methane concentrations along the north transect were lower than in the other three transects, and surface methane concentrations were mostly in equilibrium with the atmosphere except for two locations (Figure 3), indicating that methane was being transported from nearby seafloor emissions by ocean currents. A similar result has been observed at $20 \mathrm{~m}$ water depth in the Coal Oil Point seep field near Santa Barbara, California [39].

4.2. Controls on Methane Distribution. Owing to the shallow depths $(<20 \mathrm{~m})$ and strong tidal currents, thermal or haline stratification does not occur in the Lingtou Promontory seep area because there are no salinity and temperature gradients between the surface and bottom waters (Figure 4). Due to the strong tidal currents and ocean currents, the dissolved $\mathrm{O}_{2}$ concentrations remain in approximate equilibrium with atmospheric concentrations, with no gradients between the surface and bottom waters (Figure 4). In addition, the dissolved methane concentrations are not correlated with dissolved $\mathrm{O}_{2}$ concentration, salinity, and temperature (Figure 4). Although dissolved methane concentrations in bottom waters were statistically higher than that in surface waters, the difference (on average $\sim 48.8 \%$ ) was much larger than that in the nearshore shallow coast area of the North Sea (on average $14 \%$ ) [43], because gas bubbles are transported a long distance by ocean currents when they have risen to the sea surface. Hence, on account of the shallow depths and well-mixed water column, there is a loss of methane between the bottom and surface waters, unlike in deeper and stratified areas such as Tommeliten and south of the Dogger Bank [43].

The observed bubble emissions are responsible for generating the gas plume in the Lingtou Promontory (Figure 1). The methane maxima above the seafloor observed in the vertical profiles indicate a gaseous methane input from the seabed. Furthermore, the fact that methane maxima were observed above the seafloor suggests gas deposition from bubble dissolution. When gas bubbles rise through the water column, they expand because of the decrease in hydrostatic pressure and at the same time decrease in volume because of dissolution [48]. Owing to the dominating effect of the decrease in bubble size, the net buoyancy force decreases during ascent, and methane is deposited at water depths where hydrostatic equilibrium is reached and bubbles cannot continue moving. However, on account of the shallow water depths, the gas bubbles $(d=1-2 \mathrm{~cm})$ contain approximately $95 \%$ methane on reaching the sea surface [24]. Therefore, there is little loss of methane from gas bubbles as they ascend from the bottom to the surface.

4.3. Air-Sea Methane Flux. Air-sea methane emission fluxes were estimated for water sampled at $0.5 \mathrm{~m}$ water depth in the surface mixed layer. The estimated fluxes range between 1.4 and $13058.44 \mu \mathrm{mol} \mathrm{m}{ }^{-2} \mathrm{~d}^{-1}$ in the nearshore Lingtou Promontory (Figure 5). The median value is $672.57 \mu \mathrm{mol} \mathrm{m}^{-2} \mathrm{~d}^{-1}$, which is approximately four times the reported rate of methane emission in Santa Barbara Channel (Coal Oil Point; $180 \mu \mathrm{mol} \mathrm{m}^{-2} \mathrm{~d}^{-1}$ ), one of the 

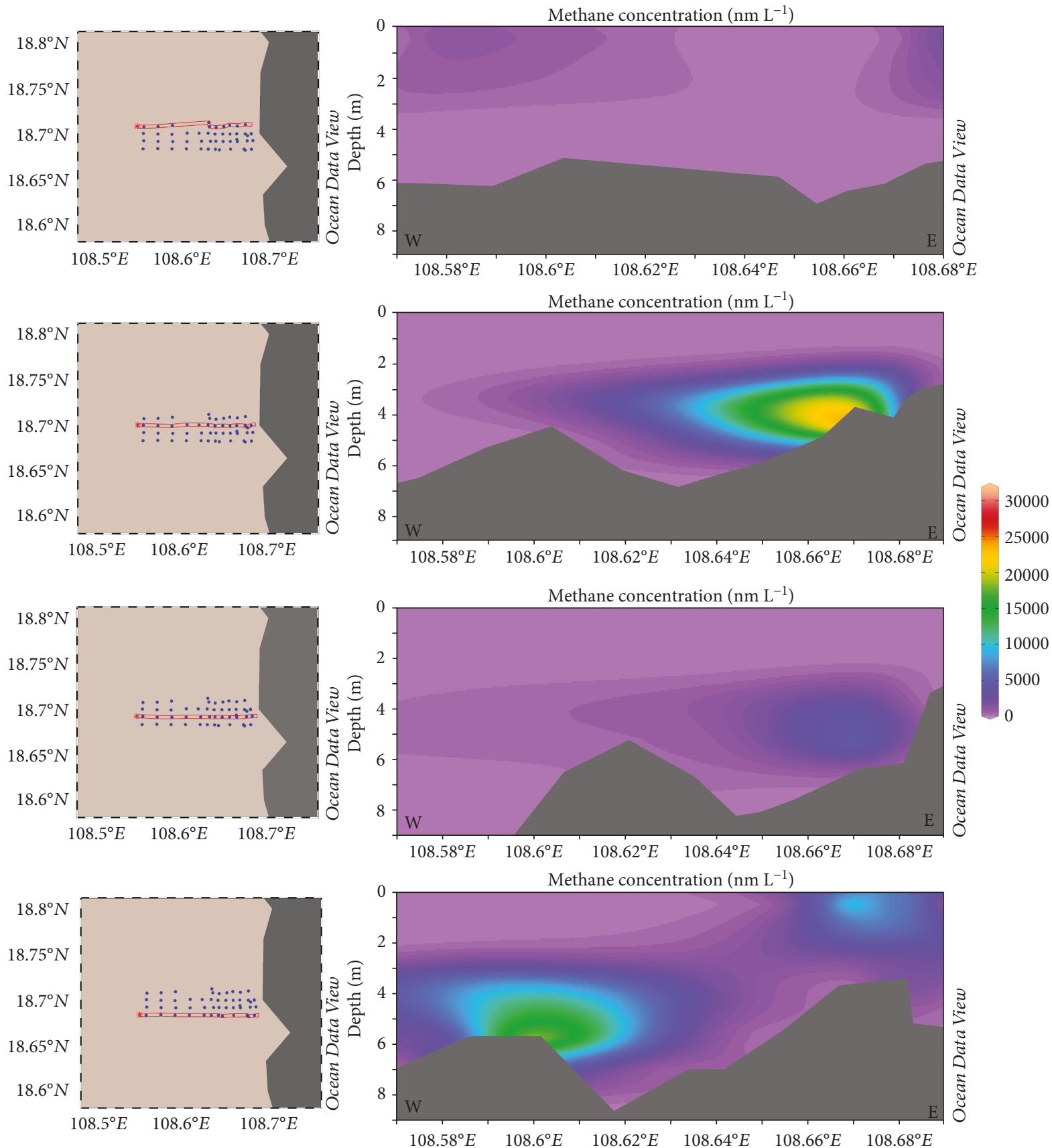

Figure 3: East-west transects of dissolved methane concentrations at the Lingtou Promontory seep area. The red stars are the starting point.

most active marine seep areas in the world [39]. The mean value is 18 times higher than the global average flux values for continental shelves (21.6 to $36.29 \mu \mathrm{mol} \mathrm{m}{ }^{-2} \mathrm{~d}^{-1}$ [26]) and four orders of magnitude higher than values characteristic of the open ocean $\left(0.2-0.5 \mu \mathrm{mol} \mathrm{m}{ }^{-2} \mathrm{~d}^{-1}\right)$ [49]. However, it has been estimated that $0.807 \times 10^{4}$ to 1.14 $\times 10^{4} \mathrm{~m}^{3} \mathrm{yr}^{-1}$ of methane is emitted from hydrocarbon seeps $[29,30]$. The total air-sea flux of methane in the nearshore Lingtou Promontory seep area is negligible compared with the flux of the methane bubbles. By analogy with the Lingtou Promontory seep area, other coastal regions of the Yinggehai Basin, such as the Yinggehai riv- ulet mouth, Yazhou Bay, the Nanshan Promontory, and the Tianya Promontory (shallower than $50 \mathrm{~m}$ ), may emit more than $672.57 \mu \mathrm{mol} \mathrm{m}{ }^{-2} \mathrm{~d}^{-1}$ of methane to the atmosphere [28]. Therefore, the coastal regions of the Yinggehai Basin in the hydrocarbon seep areas are significant sources of methane to the atmosphere, and the air-sea methane fluxes were probably underestimates.

4.4. Methane Source. Methane-rich fluids forming gas flares at the seafloor migrate along faults and/or stratigraphic boundaries. Orange et al. [50] and Forrest et al. [51] have shown that the linear arrangement of hydrocarbon seeps on 


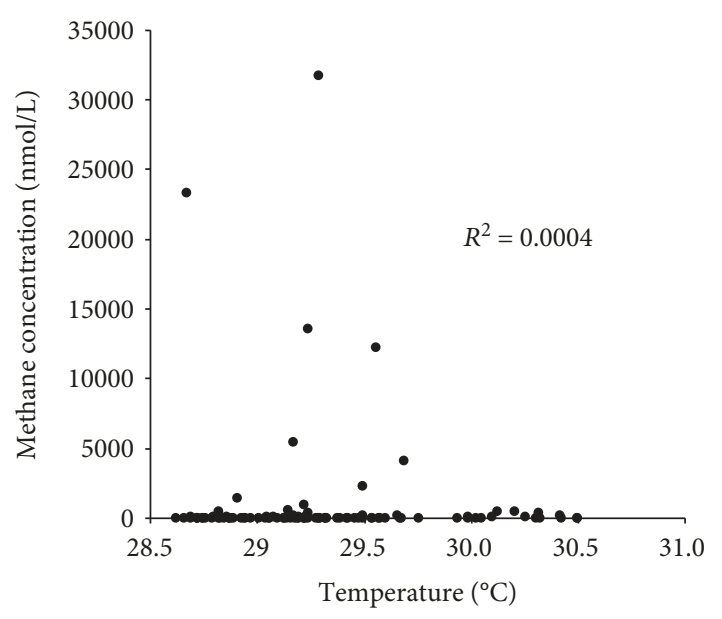

(a)

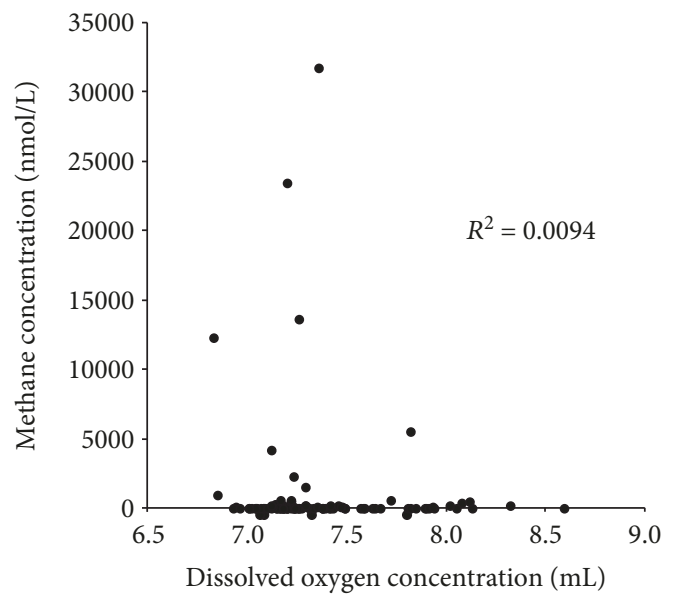

(c)

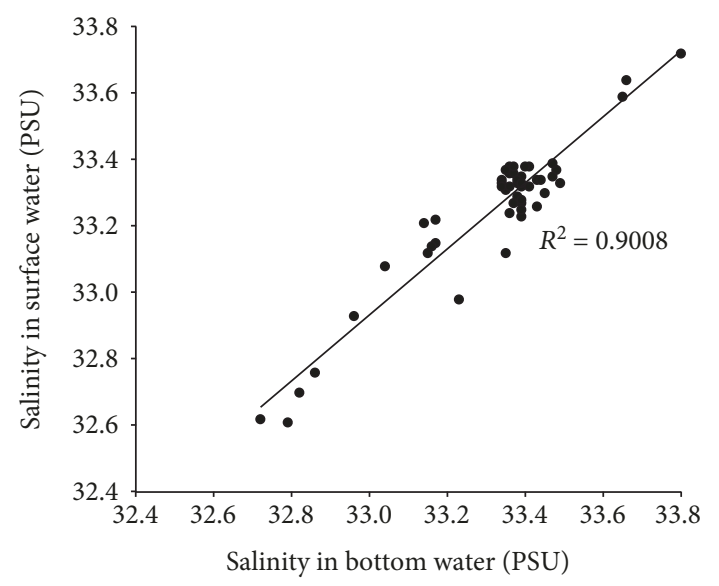

(e)

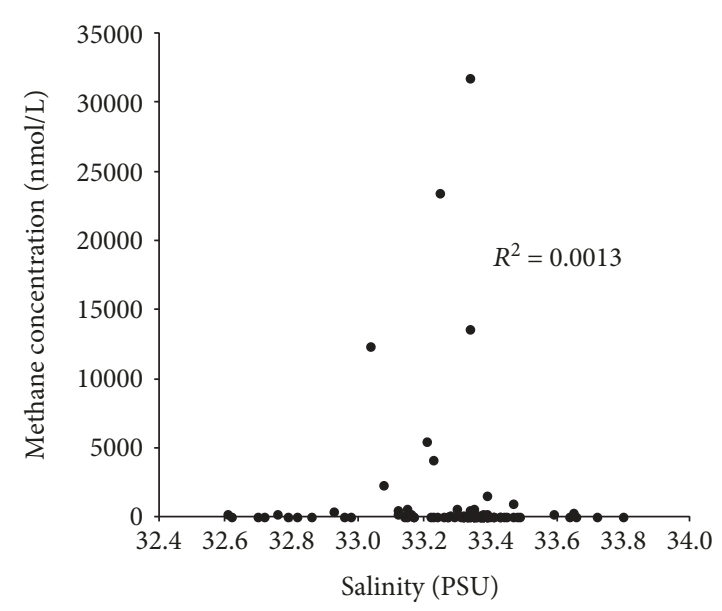

(b)

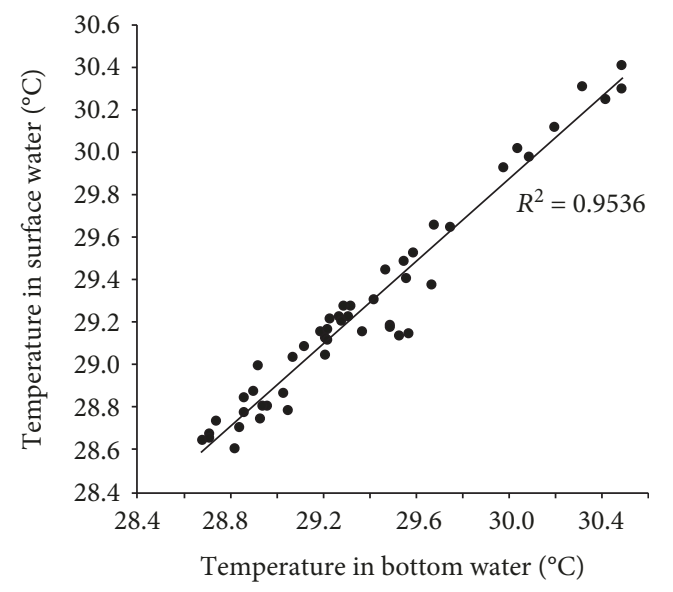

(d)

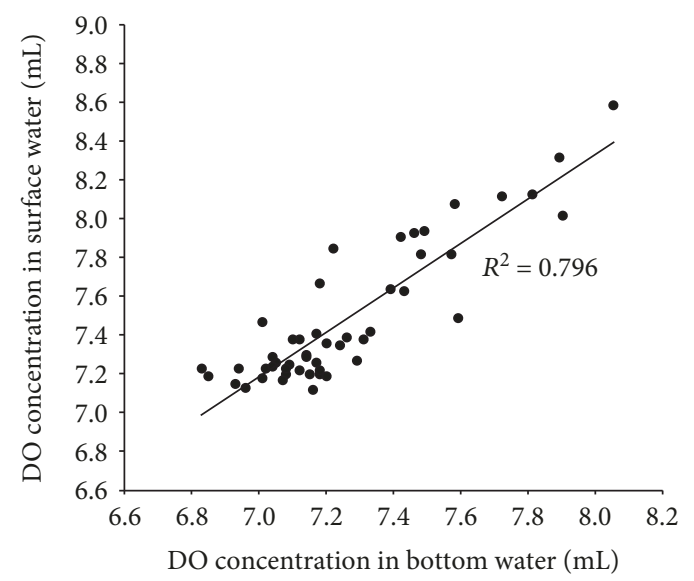

(f)

FIGURE 4: Dissolved methane concentrations in relation to dissolved oxygen concentration (a), salinity (b), and temperature (c) and comparison of temperature, salinity, and dissolved $\mathrm{O}_{2}$ concentration in surface and bottom water $(\mathrm{d}-\mathrm{f})$.

the seabed is controlled by a neighboring fault. All gas bubble flares located along the shore of Hanna Island are in the vicinity of the No. 1 Fault $[28,35]$. Generally, anomalously high methane concentrations in seawater are associated with hydrocarbon seeps, such as the COP seep area and the western Spitsbergen shelf, which are in turn related to adjacent faults [39, 52-55]. In the Lingtou Promontory seep area, we further suggest that the sandy sediments facilitate the migration of methane-rich fluid to the seafloor. Hence, we assume fluid migration along structural pathways, particularly the No. 1 Fault. In addition, according to the seismic profile of the central diapir zone in the Yinggehai Basin, methane- 


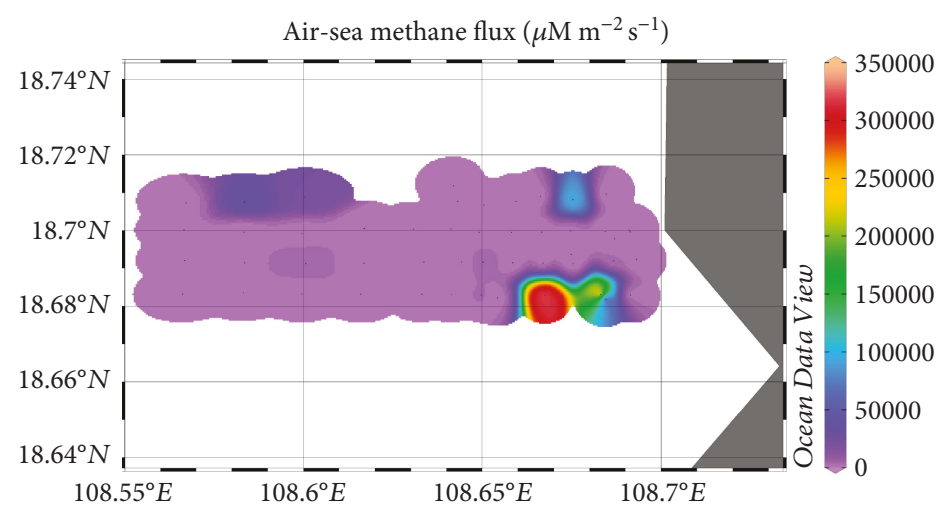

Figure 5: The air-sea flux of methane at the Lingtou Promontory seep area. The average wind speed in this area was $5.1 \mathrm{~m} \mathrm{~s}^{-1}$.

rich fluids may be expelled by mud diapirs and migrate along the dipping strata [56]. A similar phenomenon was also reported for the offshore Prins Karls Forland at $\sim 250 \mathrm{~m}$ water depth [57].

To further constrain the sources of methane, we measured the stable carbon isotopic composition of dissolved methane in four water samples with high methane concentrations that had been collected from Lingtou Promontory seep area. The $\delta^{13} \mathrm{C}_{\mathrm{CH}_{4}}$ values of two of these samples were $-59.76 \%$ and $-48.59 \%$ o (Table 1). The fact that $\delta^{13} \mathrm{C}_{\mathrm{CH}_{4}}$ values in a sea-surface and sea-sediment interface sample are similar suggests that aerobic microbial oxidation is negligible in shallow waters $(<10 \mathrm{~m})$.

The $\delta^{13} \mathrm{C}_{\mathrm{CH}_{4}}$ values of gas bubbles collected from seeps and from the sea surface in the study area are $-35.98 \%$ o and $-35.51 \%$, respectively (Table 1 ) $[28,35]$. These $\delta^{13}$ $\mathrm{C}_{\mathrm{CH}_{4}}$ values are distinctly higher than those measured in seawater, which reflects the mixing of methane from gas bubbles with biogenic methane generated in sediments. Without background $\delta^{13} \mathrm{C}_{\mathrm{CH}_{4}}$ values from the bottom and the surface ocean, we could not fully establish the mixing fraction of the methane. Notably, we were able to smell sulfurous odors in sediments collected in the shallow waters of the Lingtou Promontory seep area. It is concluded that the methane originally discharged at the seafloor was oxidized before sampling. However, we do not discount the possibility that the methane could have been removed by migration or preferential microbial oxidation $[58,59]$.

\section{Conclusions}

We observed high spatial variability in dissolved methane concentrations in surface and bottom waters in the Lingtou Promontory seep area. Dissolved methane concentrations in surface waters and bottom waters range from 2.90 to $13577.35 \mathrm{nmol} \mathrm{L}^{-1}$ and from 4.98 to $31740.02 \mathrm{nmol} \mathrm{L}^{-1}$, respectively, indicating oversaturation at all stations. The persistent anomalously high methane concentrations in seawater indicate an efficient transfer of dissolved methane from the seafloor to the surface waters. Furthermore, $\delta^{13} \mathrm{C}$ values of the dissolved methane in the studied seawater range from
$-59.76 \%$ o to $-48.59 \%$, indicating a mixed thermogenic and biogenic methane from the sediment. The measured average methane flux $\left(\sim 672.57 \mu \mathrm{mol} \mathrm{m}{ }^{-2} \mathrm{~s}^{-1}\right)$ is much higher than values characteristic of continental shelves. It is suggested that the coastal regions of Yinggehai Basin may emit more than $672.57 \mu \mathrm{mol} \mathrm{m}^{-2} \mathrm{~d}^{-1}$ of methane to the atmosphere. Coastal regions, especially shallow hydrocarbon seep areas, on the continental margin may therefore be an important hot spot of methane emissions to the atmosphere.

\section{Data Availability}

The data used to support the findings of this study are available from the corresponding author upon request.

\section{Conflicts of Interest}

The authors declare that there are no conflicts of interest regarding the publication of this paper.

\section{Acknowledgments}

We thank Dr. Baojia Huang for providing the coordinates of the gas seeps. This study was partially supported by the National Key R\&D Program of China (2017YFC0307704) and the National Natural Science Foundation of China (41676046 and 41730528).

\section{References}

[1] D. A. Stolper, M. Lawson, C. L. Davis et al., "Formation temperatures of thermogenic and biogenic methane," Science, vol. 344, no. 6191, pp. 1500-1503, 2014.

[2] A. Wilhelms, S. R. Larter, I. Head, P. Farrimond, R. di-Primio, and C. Zwach, "Biodegradation of oil in uplifted basins prevented by deep-burial sterilization," Nature, vol. 411, no. 6841, pp. 1034-1037, 2001.

[3] IPCC, Climate Change 2013 - The Physical Science Basis Contribution of Working Group I to the Fifth Assessment Report of the Intergovernmental Panel on Climate Change, Cambridge University Press, 2013.

[4] IPCC, IPCC fourth assessment report (AR4), in Climate Change 2007: The Physical Science Basis. Contribution of Working Group I to the Fourth Assessment Report of the 
Intergovernmental Panel on Climate Change, Cambridge University Press, 2007.

[5] G. Rehder, R. S. Keir, E. Suess, and T. Pohlmann, "The multiple sources and patterns of methane in North Sea waters," Aquatic Geochemistry, vol. 4, no. 3/4, pp. 403-427, 1998.

[6] V. Ramaswamy, O. Boucher, J. Haigh et al., "Radiative forcing of climate change," in Climate Change, pp. 349-416, Cambridge University Press, Cambridge, UK, 2001.

[7] M. Hovland, A. G. Judd, and R. A. Burke Jr., "The global flux of methane from shallow submarine sediments," Chemosphere, vol. 26, no. 1-4, pp. 559-578, 1993.

[8] G. Etiope, "Natural emissions of methane from geological seepage in Europe," Atmospheric Environment, vol. 43, no. 7, pp. 1430-1443, 2009.

[9] A. G. Judd, M. Hovland, L. I. Dimitrov, S. Garcia Gil, and V. Jukes, "The geological methane budget at continental margins and its influence on climate change," Geofluids, vol. 2, no. 2, 126 pages, 2002.

[10] A. G. Judd, "Natural seabed gas seeps as sources of atmospheric methane," Environmental Geology, vol. 46, no. 8, pp. 988-996, 2004.

[11] D. Wuebbles and K. Hayhoe, "Atmospheric methane: trends and impacts," in Non-CO Greenhouse Gases: Scientific Understanding, Control and Implementation, pp. 1-44, Springer, Netherlands, 2000.

[12] D. J. Wuebbles and K. Hayhoe, "Atmospheric methane and global change," Earth-Science Reviews, vol. 57, no. 3-4, pp. 177-210, 2002.

[13] W. S. Reeburgh, "Oceanic methane biogeochemistry," Chemical Reviews, vol. 107, no. 2, pp. 486-513, 2007.

[14] A. Boetius, K. Ravenschlag, C. J. Schubert et al., "A marine microbial consortium apparently mediating anaerobic oxidation of methane," Nature, vol. 407, no. 6804, pp. 623-626, 2000.

[15] K. Knittel and A. Boetius, "Anaerobic oxidation of methane: progress with an unknown process," Annual Review of Microbiology, vol. 63, no. 1, pp. 311-334, 2009.

[16] P. Regnier, A. W. Dale, S. Arndt, D. E. LaRowe, J. Mogollón, and P. van Cappellen, "Quantitative analysis of anaerobic oxidation of methane (AOM) in marine sediments: a modeling perspective," Earth-Science Reviews, vol. 106, no. 1-2, pp. 105-130, 2011.

[17] K. A. Campbell, "Hydrocarbon seep and hydrothermal vent paleoenvironments and paleontology: past developments and future research directions," Palaeogeography, Palaeoclimatology, Palaeoecology, vol. 232, no. 2-4, pp. 362-407, 2006.

[18] A. Judd and M. Hovland, Submarine Fluid Flow, the Impact on Geology, Biology, and the Marine Environment, Cambridge University Press, 2007.

[19] L. Naudts, J. Greinert, J. Poort et al., "Active venting sites on the gas-hydrate-bearing Hikurangi Margin, off New Zealand: diffusive- versus bubble-released methane," Marine Geology, vol. 272, no. 1-4, pp. 233-250, 2010.

[20] S. Mau, M. B. Heintz, and D. L. Valentine, "Quantification of $\mathrm{CH}_{4}$ loss and transport in dissolved plumes of the Santa Barbara Channel, California," Continental Shelf Research, vol. 32, pp. 110-120, 2012.

[21] M. D. Tryon, K. M. Brown, and M. E. Torres, "Fluid and chemical flux in and out of sediments hosting methane hydrate deposits on Hydrate Ridge, OR, II: hydrological processes,"
Earth and Planetary Science Letters, vol. 201, no. 3-4, pp. 541-557, 2002.

[22] J. Greinert and D. F. McGinnis, "Single bubble dissolution model-the graphical user interface SiBu-GUI," Environmental Modelling \& Software, vol. 24, no. 8, pp. 10121013, 2009.

[23] P. Linke, S. Sommer, L. Rovelli, and D. F. McGinnis, "Physical limitations of dissolved methane fluxes: the role of bottomboundary layer processes," Marine Geology, vol. 272, no. 1-4, pp. 209-222, 2010.

[24] D. F. McGinnis, J. Greinert, Y. Artemov, S. E. Beaubien, and A. Wüest, "Fate of rising methane bubbles in stratified waters: how much methane reaches the atmosphere?," Journal of Geophysical Research, vol. 111, no. C9, article C09007, 2006.

[25] G. Rehder, R. W. Collier, K. Heeschen, P. M. Kosro, J. Barth, and E. Suess, "Enhanced marine $\mathrm{CH}_{4}$ emissions to the atmosphere off Oregon caused by coastal upwelling," Global Biogeochemical Cycles, vol. 16, no. 3, pp. 2-1-2-11, 2002.

[26] H. W. Bange, U. H. Bartell, S. Rapsomanikis, and M. O. Andreae, "Methane in the Baltic and North seas and a reassessment of the marine emissions of methane," Global Biogeochemical Cycles, vol. 8, no. 4, pp. 465-480, 1994.

[27] R. C. Upstill-Goddard, J. Barnes, T. Frost, S. Punshon, and N. J. P. Owens, "Methane in the southern North Sea: lowsalinity inputs, estuarine removal, and atmospheric flux," Global Biogeochemical Cycles, vol. 14, no. 4, pp. 1205-1217, 2000.

[28] B. Huang, X. Xiao, X. Li, and D. Cai, "Spatial distribution and geochemistry of the nearshore gas seepages and their implications to natural gas migration in the Yinggehai Basin, offshore South China Sea," Marine and Petroleum Geology, vol. 26, no. 6, pp. 928-935, 2009.

[29] P. Di, D. Feng, and D. Chen, "In-situ and on-line measurement of gas flux at a hydrocarbon seep from the northern South China Sea," Continental Shelf Research, vol. 81, pp. 80-87, 2014.

[30] P. Di, D. Feng, and D. Chen, "Temporal variation in natural gas seep rate and influence factors in the Lingtou promontory seep field of the northern South China Sea," Terrestrial, Atmospheric and Oceanic Sciences, vol. 25, no. 5, pp. 665-672, 2014.

[31] B. Huang, X. Xiao, and X. Li, "Geochemistry and origins of natural gases in the Yinggehai and Qiongdongnan Basins, offshore South China Sea," Organic Geochemistry, vol. 34, no. 7, pp. 1009-1025, 2003.

[32] B. Huang, X. Xiao, and W. Zhu, "Geochemistry, origin, and accumulation of $\mathrm{CO}_{2}$ in natural gases of the Yinggehai Basin, offshore South China Sea," AAPG Bulletin, vol. 88, no. 9, pp. 1277-1293, 2004.

[33] B. Huang, X. Xiao, Z. Hu, and P. Yi, "Geochemistry and episodic accumulation of natural gases from the Ledong gas field in the Yinggehai Basin, offshore South China Sea," Organic Geochemistry, vol. 36, no. 12, pp. 1689-1702, 2005.

[34] P. Di, Q. Chen, and D. Chen, "Quantification of methane fluxes from hydrocarbon seeps to the ocean and atmosphere: development of an in situ and online gas flux measuring system," Journal of Ocean University of China, vol. 16, no. 3, pp. 447-454, 2017.

[35] P. Di, D. Feng, and D. Chen, "The distribution and variation in the gas composition of macro-seeps on the near-shore Lingtou Promontory in the South China Sea," Acta Oceanologica Sinica, vol. 35, no. 11, pp. 120-125, 2016. 
[36] A. H. Knap, A. E. Michaels, A. Close, H. Ducklow, and A. Dickson, "Protocols for the Joint Global Ocean Flux Study (JGOFS) core measurements," JGOFS Report Nr. 19, 1996.

[37] D. L. Valentine, D. C. Blanton, W. S. Reeburgh, and M. Kastner, "Water column methane oxidation adjacent to an area of active hydrate dissociation, Eel river Basin," Geochimica et Cosmochimica Acta, vol. 65, no. 16, pp. 2633-2640, 2001.

[38] K. M. Johnson, J. E. Hughes, P. L. Donaghay, and J. M. Sieburth, "Bottle -calibration static head space method for the determination of methane dissolved in seawater," Analytical Chemistry, vol. 62, no. 21, pp. 2408-2412, 1990.

[39] I. Leifer and I. MacDonald, "Dynamics of the gas flux from shallow gas hydrate deposits: interaction between oily hydrate bubbles and the oceanic environment," Earth and Planetary Science Letters, vol. 210, no. 3-4, pp. 411-424, 2003.

[40] D. A. Wiesenburg and N. L. Guinasso, "Equilibrium solubilities of methane, carbon monoxide, and hydrogen in water and sea water," Journal of Chemical \& Engineering Data, vol. 24, no. 4, pp. 356-360, 1979.

[41] R. Wanninkhof, W. E. Asher, D. T. Ho, C. Sweeney, and W. R. McGillis, "Advances in quantifying air-sea gas exchange and environmental forcing," Annual Review of Marine Science, vol. 1, no. 1, pp. 213-244, 2009.

[42] R. Wanninkhof, "Relationship between wind speed and gas exchange over the ocean revisited," Limnology and Oceanography: Methods, vol. 12, no. 6, pp. 351-362, 2014.

[43] A. V. Borges, W. Champenois, N. Gypens, B. Delille, and J. Harlay, "Massive marine methane emissions from nearshore shallow coastal areas," Scientific Reports, vol. 6, no. 1, article 27908, 2016.

[44] Z. Duan and S. Mao, "A thermodynamic model for calculating methane solubility, density and gas phase composition of methane-bearing aqueous fluids from 273 to $523 \mathrm{~K}$ and from 1 to 2000 bar," Geochimica et Cosmochimica Acta, vol. 70, no. 13, pp. 3369-3386, 2006.

[45] S. Mau, M. Römer, M. E. Torres et al., "Widespread methane seepage along the continental margin off Svalbard - from Bjørnøya to Kongsfjorden," Science Reports, vol. 7, no. 1, article 42997, 2017.

[46] S. Mau, T. Gentz, J. H. Körber et al., "Seasonal methane accumulation and release from a gas emission site in the central North Sea," Biogeosciences, vol. 12, no. 18, pp. 5261-5276, 2015.

[47] J. A. Screen and I. Simmonds, "The central role of diminishing sea ice in recent Arctic temperature amplification," Nature, vol. 464, no. 7293, pp. 1334-1337, 2010.

[48] I. Leifer and R. K. Patro, "The bubble mechanism for methane transport from the shallow sea bed to the surface: a review and sensitivity study," Continental Shelf Research, vol. 22, no. 16, pp. 2409-2428, 2002.

[49] T. S. Rhee, A. J. Kettle, and M. O. Andreae, "Methane and nitrous oxide emissions from the ocean: a reassessment using basin-wide observations in the Atlantic," Journal of Geophysical Research, vol. 114, no. D12, article D12304, 2009.

[50] D. L. Orange, H. G. Greene, D. Reed et al., "Widespread fluid expulsion on a translational continental margin: mud volcanoes, fault zones, headless canyons, and organic-rich substrate in Monterey Bay, California," Geological Society of America Bulletin, vol. 111, no. 7, pp. 992-1009, 1999.
[51] M. J. Forrest, J. Ledesma-Vázquez, W. Ussler III, J. T. Kulongoski, D. R. Hilton, and H. G. Greene, "Gas geochemistry of a shallow submarine hydrothermal vent associated with the El Requesón fault zone, Bahía Concepción, Baja California Sur, México," Chemical Geology, vol. 224, no. 1-3, pp. 82-95, 2005.

[52] J. Knies, E. Damm, J. Gutt, U. Mann, and L. Pinturier, "Near-surface hydrocarbon anomalies in shelf sediments off Spitsbergen: evidences for past seepages," Geochemistry, Geophysics, Geosystems, vol. 5, no. 6, 2004.

[53] E. Damm, A. Mackensen, G. Budéus, E. Faber, and C. Hanfland, "Pathways of methane in seawater: plume spreading in an Arctic shelf environment (SW-Spitsbergen)," Continental Shelf Research, vol. 25, no. 12-13, pp. 1453-1472, 2005.

[54] I. Leifer and D. Culling, "Formation of seep bubble plumes in the Coal Oil Point seep field," Geo-Marine Letters, vol. 30, no. 3-4, pp. 339-353, 2010.

[55] S. Mau, M. B. Heintz, F. S. Kinnaman, and D. L. Valentine, "Compositional variability and air-sea flux of ethane and propane in the plume of a large, marine seep field near Coal Oil Point, CA," Geo-Marine Letters, vol. 30, no. 3-4, pp. 367$378,2010$.

[56] P. F. Di, H. G. Huang, B. J. Huang, J. X. He, and D. F. Chen, "Seabed pockmark formation associated with mud diapir development and fluid activities in the Yinggehai Basin of the South China Sea," Journal of Tropical Oceanography, vol. 31, pp. 26-36, 2012.

[57] A. Rajan, J. Mienert, and S. Bünz, “Acoustic evidence for a gas migration and release system in Arctic glaciated continental margins offshore NW-Svalbard," Marine and Petroleum Geology, vol. 32, no. 1, pp. 36-49, 2012.

[58] A. Prinzhofer and É. Pernaton, "Isotopically light methane in natural gas: bacterial imprint or diffusive fractionation," Chemical Geology, vol. 142, no. 3-4, pp. 193-200, 1997.

[59] S. D. Mendes, M. C. Redmond, K. Voigritter, C. Perez, R. Scarlett, and D. L. Valentine, "Marine microbes rapidly adapt to consume ethane, propane, and butane within the dissolved hydrocarbon plume of a natural seep," Journal of Geophysical Research: Oceans, vol. 120, no. 3, pp. 1937-1953, 2015. 

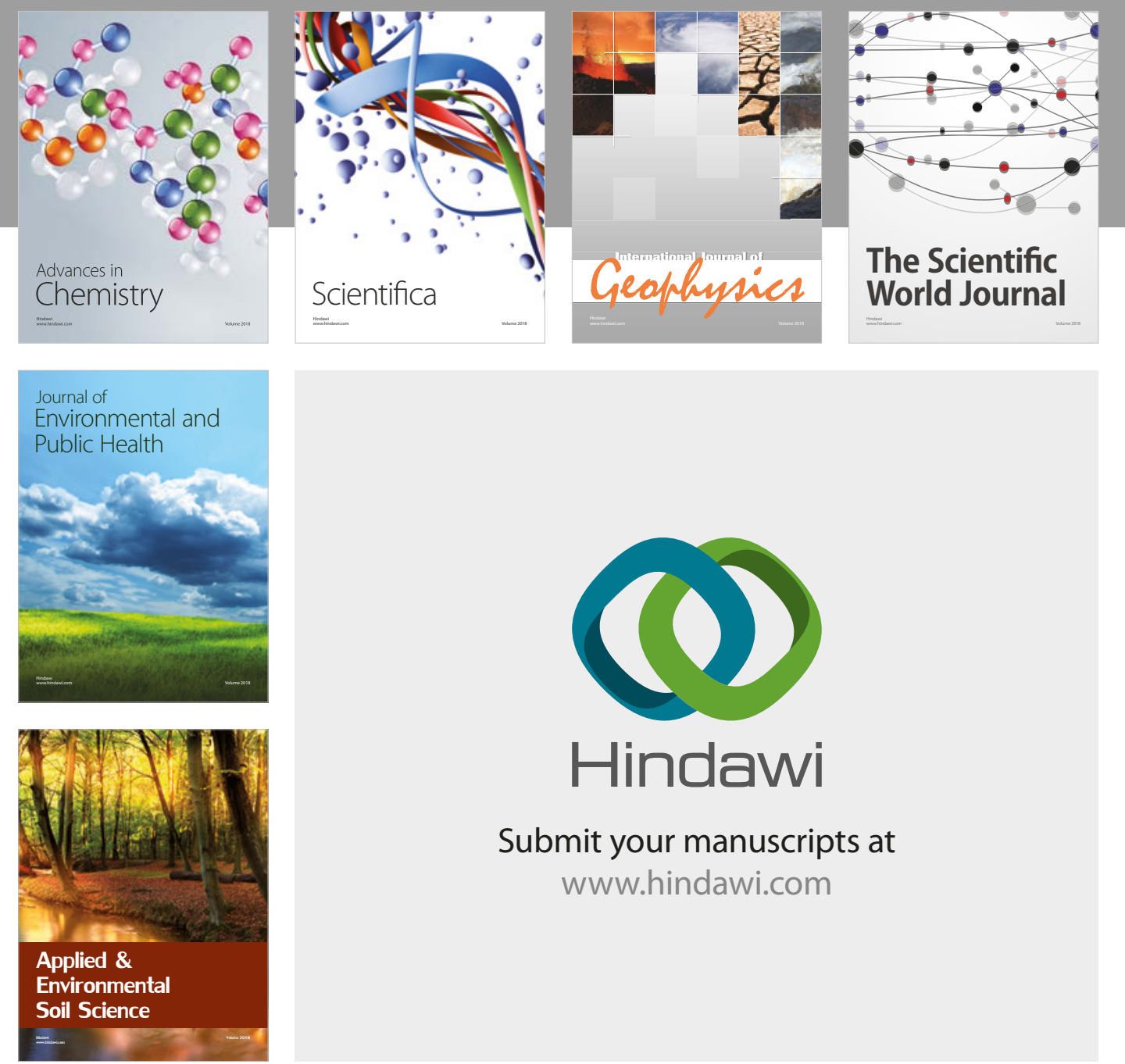

The Scientific

\section{World Journal}
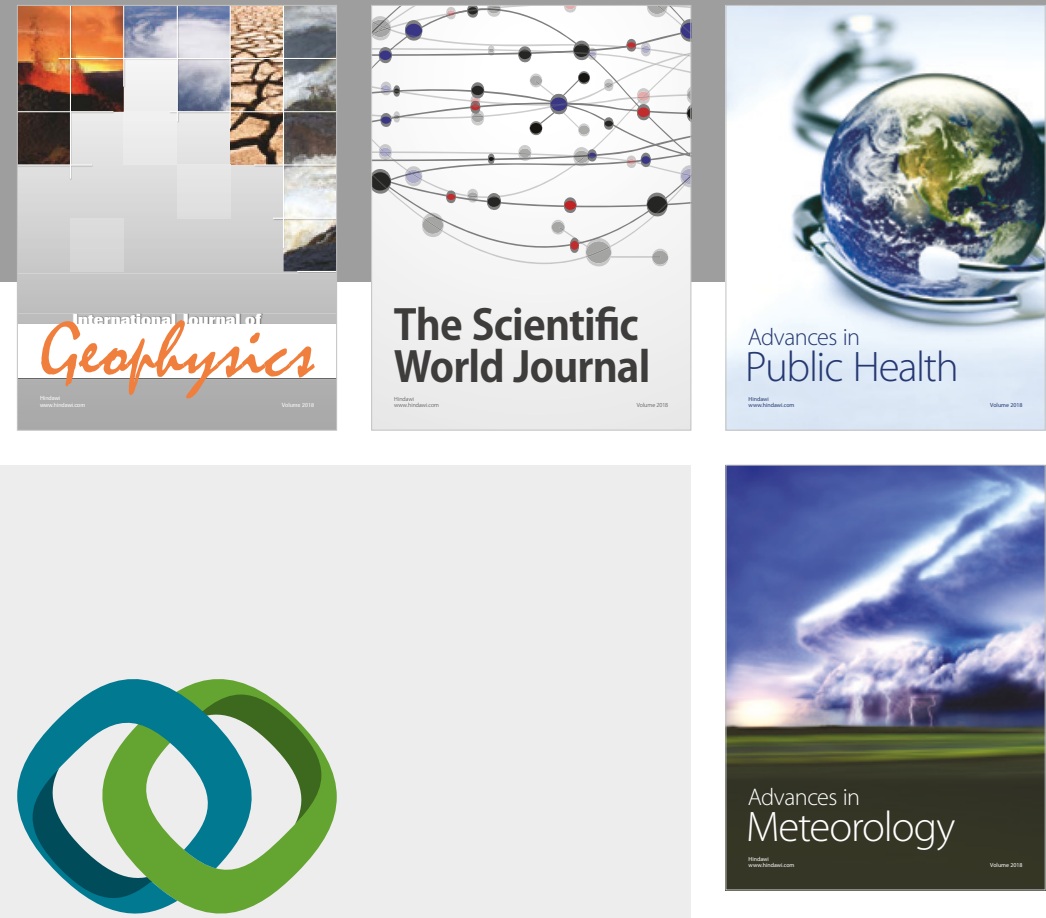

Advan

Public Health

\section{Hindawi}

Submit your manuscripts at

www.hindawi.com
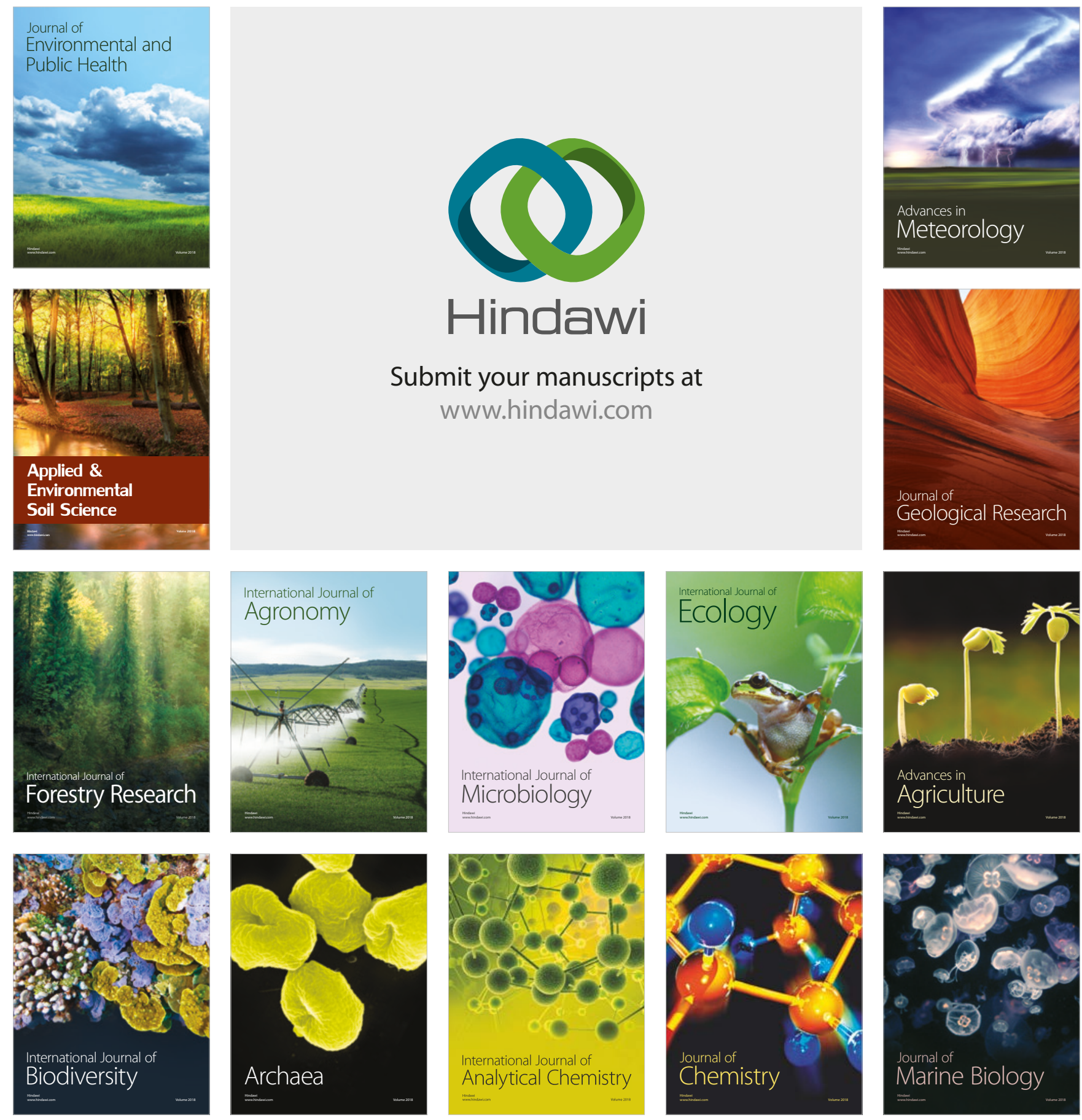